\title{
Percurso do diagnóstico de malignidades
}

\author{
Course the diagnosis of malignancies
}

Jênifer Pereira Vitoriano ${ }^{* *}$, Suelen Brito Nascimento ${ }^{\ddagger}$, Isabela Oliveira Alves da Silva ${ }^{\S}$, Sara dos Santos Nascimentoll, Hye Chung Kang ${ }^{\mathrm{O}}$

Como citar esse artigo. Vitoriano, J.P.;

Nascimento, S.B..; da Silva, I.O.A.;

Nascimento, S.S.; Kang, H.C. Percurso do diagnóstico de malignidades. Revista de Saúde. 2021 Dez./Mar.; 12 (1): 48-54.

\section{Resumo}

O câncer é responsável por aproximadamente 9,6 milhões de mortes anuais no mundo, as malignidades hematológicas ocupam mais de 7\% desse total. Diagnosticá-las é um trabalho complexo e parte da falha na quimioterapia decorre do tratamento tardio. Este trabalho propõe uma reflexão sobre a importância de encurtar o processo de diagnóstico de neoplasias e seu impacto no prognóstico/tratamento. Foram analisados trabalhos que abordavam o tempo desde os primeiros sintomas até o diagnóstico dos pacientes que apresentavam alguma malignidade, especialmente hematológicas. A revisão foi realizada consultando as bases Scielo e PubMed (intervalo 2015-2019). Também, foi realizada pesquisa sobre a mesma temática no Hospital Universitário Antônio Pedro, por meio de questionário aplicado aos pacientes que frequentavam o ambulatório de quimioterapia. Foram identificadas 276 publicações no PubMed e 44 no Scielo e selecionados 7 artigos, somente um abordava malignidades hematológicas. Os resultados referentes ao questionário incluem 39 pacientes (71,80\% mulheres; 28,20\% homens), dentre os quais, os portadores de neoplasias hematológicas representam 41,02\%. A busca pelo atendimento médico após o início dos sintomas foi feita por 48,72\% dos indivíduos, $38,46 \%$ tiveram diagnóstico em estágio avançado. O serviço inicial com maior procura foi a Unidade Básica de Saúde $(51,29 \%)$. Os sintomas mais relatados foram dores e cansaço $(41,00 \%)$. A idade média foi 55 anos. Tanto a revisão da literatura quanto a pesquisa no HUAP evidenciaram um atraso significativo na busca pelo diagnóstico, consequentemente, do tratamento, o que pode resultar em resposta insatisfatória podendo evoluir a óbito.

Palavras-chave: Câncer; Malignidades Hematológicas; Atenção Primária; Prognóstico.

\begin{abstract}
ICancer is responsible for approximately 9.6 million annual deaths worldwide, hematological malignancies occupy more than $7 \%$ of this total. Diagnosing them is a complex job and part of the failure in chemotherapy is due to late treatment. This work proposes a reflection on the importance of shortening the process of diagnosis of neoplasms and its impact on the prognosis / treatment. Studies that analyzed the time from the first symptoms to the diagnosis of patients with some malignancy, especially hematological, were analyzed. The review was carried Scielo and PubMed databases (interval 2015-2019). Research on the same theme was also carried out at Hospital Universitário Antonio Pedro, using a questionnaire applied to patients who attended the chemotherapy outpatient clinic. 276 publications were identified in PubMed and 44 in Scielo and 7 articles were selected, only one addressed hematological malignancies. The results for the questionnaire include 39 patients $(71.80 \%$ women; $28.20 \%$ men) have been included, among which, patients with hematological neoplasms represent $41.02 \%$. The search for medical care after the onset of symptoms was made by $48.72 \%, 38.46 \%$ had an advanced diagnosis. The initial service with the greatest demand was the Basic Health Unit (51.29\%). The most reported symptoms were pain and tiredness (41.00\%). The average age was 55 years. Both the literature review and the research at HUAP showed a significant delay in the search for diagnosis, consequently, treatment, which can result in an unsatisfactory response and may evolve to death.
\end{abstract}

Keywords: Cancer; Hematological Malignancies; Primary Attention; Prognosis.

\section{Introdução}

O câncer tem sido considerado um grave problema de saúde pública, devido à alta incidência e complexidade de subdivisões nos diagnósticos. ${ }^{1}$ As neoplasias malignas são responsáveis por pouco mais de $15 \%$ de todas as mortes no Brasil, com cerca de 600 mil novos casos por ano estimados pelo Instituto Nacional do Câncer (INCA) em 2016², e no mundo são cerca de 9,6 milhões de óbitos anuais.3 Ainda, a estimativa para o Brasil, de acordo com o INCA, para cada ano do triênio 2020-2022 é de 625 mil casos novos de câncer. ${ }^{3}$

As neoplasias malignas são subdivididas em tumores sólidos e neoplasias hematológicas. ${ }^{1,4}$ Especificamente, as neoplasias hematológicas, malignidades que afetam o tecido sanguíneo e podem ser de origem mieloide ou linfoide, apresentam menor proporção de diagnósticos, aproximadamente $6,6 \% \mathrm{em}$ todo o mundo, mas são responsáveis por um número relativamente maior de óbitos, em torno de $7,1 \%,{ }^{1,5}$

\footnotetext{
Afiliação dos autores:

†"Graduanda de medicina da Universidade Federal Fluminense. Departamento de Patologia da Universidade Federal Fluminense. Niterói - RJ, Brasil.

${ }^{\ddagger}$ Mestranda Patologia da Universidade Federal Fluminense. Departamento de Patologia da Universidade Federal Fluminense. Niterói - RJ, Brasil.

${ }^{\S}$ Graduanda de farmácia da Universidade Federal Fluminense. Departamento de Patologia da Universidade Federal Fluminense. Niterói - RJ, Brasil.

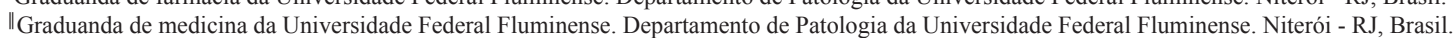

${ }^{\circ}$ Professora Doutora Patologia da Universidade Federal Fluminense. Departamento de Patologia da Universidade Federal Fluminense. Niterói - RJ, Brasil. 
que por muitas vezes ocorrem devido a um diagnóstico tardio. ${ }^{6,7}$

A maior causa para a demora no diagnóstico, está relacionada a malignidades que possuem sintomas vagos que podem ser confundidos com condições não severas. Dentre eles, podemos citar o câncer de pulmão, ${ }^{8} \mathrm{o}$ câncer colorretal, ${ }^{9}$ doenças mieloproliferativas, dentre outros. Esse hiato até o diagnóstico final do paciente pode acarretar em diagnóstico tardio, com mau prognóstico que pode evoluir para óbito.

Perante o cenário exposto, o foco principal deste trabalho foi averiguar o tempo médio que os pacientes portadores de neoplasias levaram entre os primeiros sintomas até receber o diagnóstico e iniciar o tratamento. Para tal, foram avaliados quantos trabalhos recentes há na literatura que relataram a respeito do tempo, entre os primeiros sintomas até o diagnóstico dos pacientes que apresentavam alguma malignidade, especialmente as hematológicas. Também foram examinados dados de um questionário aplicado aos pacientes que frequentavam o ambulatório de quimioterapia do Hospital Universitário Antônio Pedro (HUAP), Niterói -RJ

\section{Material e método}

Foi realizada uma revisão da literatura médica utilizando-se a metodologia descrita por Jacobsen et al. (2017). ${ }^{8}$ Foram consultadas as bases de dados científicas Scielo e Pubmed, usando "câncer" e "tempo para o diagnóstico" como palavras-chave na base de dados Scielo e "cancer" and "waiting time for diagnosis" como palavras-chave na base de dados PubMed, observandose o intervalo de tempo entre os anos 2015 a 2019 como critérios de pesquisa. Foram incluídos artigos com texto completo disponibilizados sem ônus em qualquer idioma.

Dos artigos selecionados, avaliou-se primeiramente o título de cada trabalho, relatos de caso ou títulos que abordassem avaliação da qualidade de vida do paciente após o diagnóstico, exames de imagem ou diagnóstico, pacientes pré ou pós-cirúrgicos ou que não avaliassem especificamente o tempo entre os primeiros sintomas até o diagnóstico foram excluídos.

Os títulos selecionados foram reavaliados e, posteriormente, realizou-se a revisão dos resumos e do artigo completo. Foram incluídos, em ambos os casos, apenas os trabalhos que relataram o intervalo de tempo de espera para o diagnóstico da neoplasia ou relacionado a este.

Para a coleta de dados no HUAP, foi aplicado um questionário (Anexo 1) aos pacientes diagnosticados com alguma neoplasia, atendidos pelo ambulatório de quimioterapia do HUAP da Universidade Federal Fluminense (UFF)/Empresa Brasileira de Serviços Hospitalares (EBSERH).

\section{Resultados}

Foram identificadas 276 publicações no PubMed e 44 publicações no Scielo, sendo que somente na base de dados Scielo haviam artigos em duplicata $(n=7)$, que foram excluídos, restando 37 artigos a serem analisados por título. Depois de avaliar o título dos artigos pré-

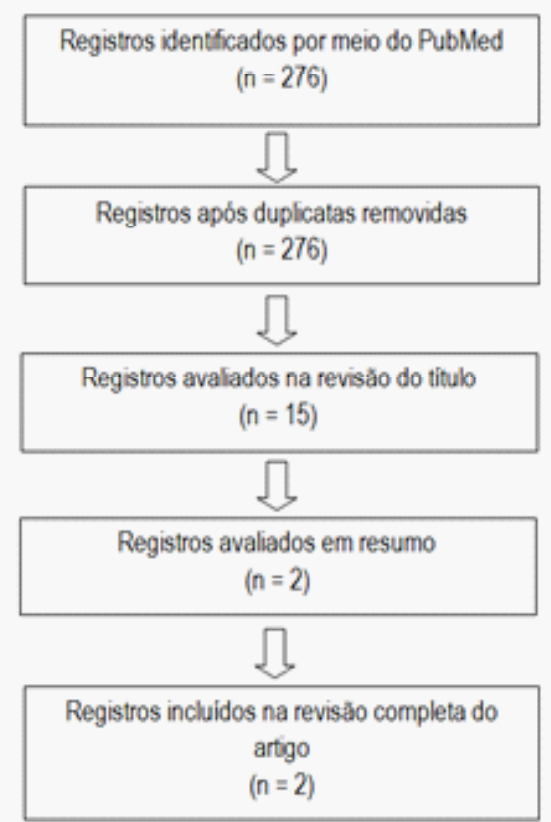

Registros excluidos ( $\mathrm{n}=261$ ): Análise da qualidade de vida pós diagnóstico/prognóstico $=62$ Pesquisas com células/mutaçöes/genes $=16$ Registrolrelato de caso $=13$

Exames de imagemidiagnóstico $=36$

Pacientes pré ou pós-cirúrgicos $=28$

Metástases $=6$

Avaliação da eficiẽncia do reastreio do câncer de mama/pâncreas/colo de útero/melanoma $=5$

Gravidez e câncer = 2

Doaçăo de órgăos/transplantes (figado/células tronco) em pacientes com câncer $=23$

Programas de reastreio de câncer (próstataligado/pulmăo/cob de útero/colorretalipele/cabeça e pescocol/mama = 31

Pesquisas com medicaçes tratamentos $=39$

Figura 1. Artigos analisados por meio da base de dados PubMed, 2019. 


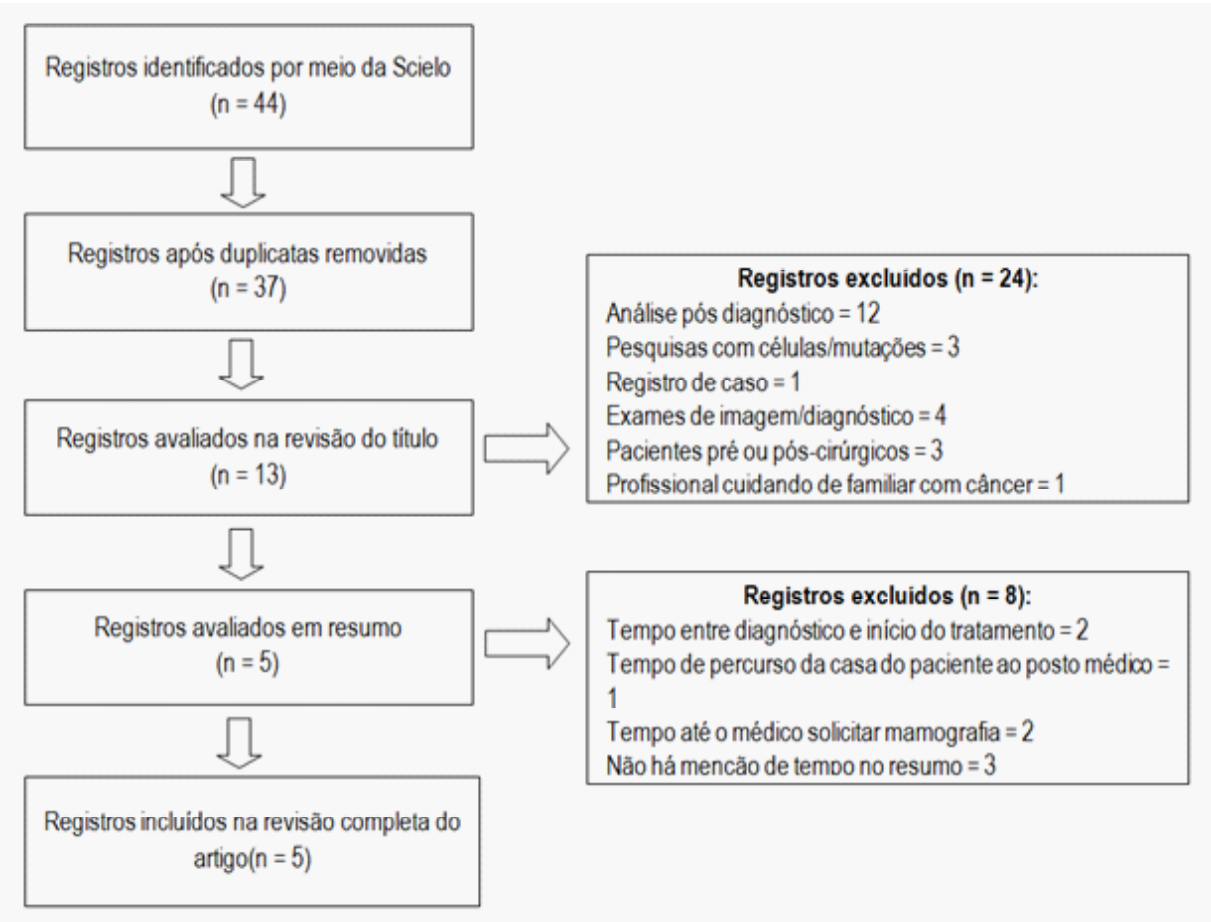

Figura 2. Artigos analisados por meio da base de dados Scielo, 2019.

\begin{tabular}{|l|l|}
\hline Scielo & PubMed \\
\hline Câncer colorretal (2016) & Câncer hematológico (2015) \\
Câncer colorretal/estômago (2017) & Câncer colorretal (2017) \\
Câncer cabeça e pescoço (2016) & \\
Câncer bucal (2016) & \\
Câncer colo de útero/próstata (2016) & \\
\hline
\end{tabular}

Figura 3. Artigos selecionados por revisão completa de texto e ano de publicação, 2019.

selecionados por meio das palavras-chave e ano de publicação, foram selecionados 15 do PubMed e 13 do Scielo para avaliação do resumo, onde elegeram-se duas publicações do PubMed e cinco publicações do Scielo para análise completa do artigo. Das publicações eleitas, todas foram incluídas na presente revisão. O número de artigos excluídos está apresentado nas Figuras 1 e 2, foram excluídos devido à ausência, no título ou no resumo, a menção à temática desta revisão.

De todos os sete artigos selecionados do PubMed e Scielo, apenas um se referia as malignidades hematológicas, enquanto três se referiam a câncer colorretal, um relatava câncer de cabeça e pescoço, um câncer bucal, e um câncer de colo de útero/próstata; observou-se também que 2016 foi o ano com maior número de publicações acerca do tema (Figura 3 ).

Na pesquisa realizada no HUAP, foram incluídos até o momento 39 pacientes, 11 homens e 28 mulheres, sendo que 20 tiveram sua suspeita clínica realizando exames de rotina. $\mathrm{O}$ tipo mais frequente de câncer foi o que acomete o tecido sanguíneo, representando 41,02\% dos pacientes, desses, $12,83 \%$ são relacionados à medula óssea. Ainda, 48,72\% dos entrevistados buscaram atendimento médico dias após o início dos sintomas, $30,77 \%$ levaram de uma a três semanas e $20,51 \%$ um mês ou mais, onde $38,46 \%$ tiveram diagnóstico em estágio avançado do câncer, $28,20 \%$ obtiveram diagnóstico no estágio inicial e 33,34 \% não souberam em qual estágio receberam o diagnóstico.

O serviço com maior procura foi a Unidade Básica de Saúde, 51,29\%, sendo que apenas um paciente deu entrada diretamente no HUAP. O sintoma mais relatado entre os pacientes foi dor e cansaço, observados em $41,00 \%$ dos casos. A média de idade dos entrevistados foi de 55 anos, tendo o paciente mais jovem 17 anos e o mais velho 78 anos. Dados mostrados na Tabela 1. 
Tabela 1. Malignidade, estágio de evolução e tempo de busca de atendimento médico de pacientes entrevistados no Hospital Universitário Antônio Pedro em 2019.

\begin{tabular}{lc}
\hline \multicolumn{1}{c}{ Variáveis } & \% de pacientes $(\mathbf{N}=\mathbf{3 9})$ \\
\hline Sexo & \\
Feminino & $71,8 \%$ \\
Masculino & $28,2 \%$ \\
Idade (média) & \\
Feminino & 56 anos \\
Masculino & 64 anos \\
Malignidade & \\
Tecido Sanguíneo & $41,02 \%$ \\
Medula Óssea & $12,83 \%$ \\
Linfoma & $5,13 \%$ \\
Laringe & $2,56 \%$ \\
Não souberam especificar & $38,46 \%$ \\
Tempo de busca de atendimento médico & \\
Dias & \\
1 a 3 semanas & $48,72 \%$ \\
1 mês ou mais & $30,77 \%$ \\
Estágio do câncer & $20,51 \%$ \\
Inicial & \\
Avançado & $28,20 \%$ \\
Não souberam o estágio & $38,46 \%$ \\
\hline
\end{tabular}

Tabela 2. Resumo dos resultados referente aos 7 trabalhos utilizados na revisão e ao estudo realizado no Hospital Universitário Antônio Pedro.

\begin{tabular}{|c|c|c|c|c|c|c|c|c|}
\hline & HUAP (2019 - atual) & $\begin{array}{l}\text { HOWELL } \\
\text { et al. } \\
(2015)\end{array}$ & $\begin{array}{c}\text { ROGERS } \\
\text { et al. } \\
(2017)\end{array}$ & $\begin{array}{c}\text { FOLETTO } \\
\text { et al. } \\
(2016)\end{array}$ & $\begin{array}{c}\text { FELIPPU } \\
\text { et al. } \\
(2016)\end{array}$ & $\begin{array}{l}\text { VALLE } \\
\text { et al. } \\
(2017)\end{array}$ & $\begin{array}{c}\text { AGUIAR } \\
\text { Jr. et al. } \\
(2016)\end{array}$ & $\begin{array}{l}\text { LE CAMPION } \\
\text { et al. } \\
(2016)\end{array}$ \\
\hline $\begin{array}{l}\text { Média de } \\
\text { idade }\end{array}$ & 55 anos & 58 anos & 50 anos & 56 anos & 63 anos & 62 anos & 54 anos & 64 anos \\
\hline Sexo $(\%)$ & $\begin{array}{c}\text { Feminino } \\
(72 \%)\end{array}$ & Não especifica & $\begin{array}{c}\text { Masculino } \\
(52 \%)\end{array}$ & $\begin{array}{c}\text { Masculino } \\
(56 \%)\end{array}$ & $\begin{array}{c}\text { Masculino } \\
(94 \%)\end{array}$ & $\begin{array}{c}\text { Masculino } \\
(53 \%)\end{array}$ & $\begin{array}{c}\text { Feminino } \\
(58 \%)\end{array}$ & $\begin{array}{c}\text { Masculino } \\
(67 \%)\end{array}$ \\
\hline Profissão & $\begin{array}{l}\text { Contato com } \\
\text { produtos de } \\
\text { limpeza }\end{array}$ & - & - & Agricultores & - & Aposentados & - & $\begin{array}{c}\text { Não } \\
\text { economicamente } \\
\text { ativos }\end{array}$ \\
\hline Sintomas & Dor e cansaço & $\begin{array}{c}\text { Fadiga, } \\
\text { linfadenopatia } \\
\text { e dor óssea }\end{array}$ & $\begin{array}{c}\text { Não } \\
\text { específica }\end{array}$ & $\begin{array}{l}\text { Dor abdominal, } \\
\text { emagrecimento e } \\
\text { sangue nas fezes }\end{array}$ & $\begin{array}{c}\text { Perda de } \\
\text { peso, dor } \\
\text { abdominal e } \\
\text { epigástrica }\end{array}$ & - & - & $\begin{array}{l}\text { Não específica, } \\
\text { lesões }\end{array}$ \\
\hline $\begin{array}{c}\text { Tempo busca } \\
\text { de } \\
\text { atendimento }\end{array}$ & Alguns dias & 3 meses & $\begin{array}{c}2 \text { a } 3 \\
\text { meses }\end{array}$ & 5 a 6 meses & 10 meses & 2 a 3 meses & 9 meses & 6 a 7 meses \\
\hline Estágio & $\begin{array}{l}\text { Inicial/Muito } \\
\text { avançado }\end{array}$ & - & Avançado & Avançado & Avançado & Inicial & Avançado & Avançado \\
\hline $\begin{array}{c}\text { Forma do } \\
\text { estudo }\end{array}$ & Entrevista & Entrevista & Entrevista & Entrevista & Entrevista & Entrevista & Entrevista & $\begin{array}{l}\text { Consulta de } \\
\text { prontuário }\end{array}$ \\
\hline Ano de estudo & 2019- & 2011 & 2008-2010 & 2012-2014 & 2011-2012 & 2014 & 2005-2015 & $2005-2013$ \\
\hline
\end{tabular}

HUAP: Hospital Universitário Antônio Pedro 
A Tabela 2 demonstra um resumo dos resultados referente aos 7 trabalhos utilizados na revisão e ao estudo realizado no Hospital Universitário Antônio Pedro.

\section{Discussão}

Todos os artigos analisados $(\mathrm{n}=7)$ coletaram os dados dos pacientes por meio de questionários e realizaram a confirmação de grande parte dessas informações por meio do acesso ao prontuário dos pacientes, sendo que somente o estudo de Le Campion et al. ${ }^{10}$ coletou os dados exclusivamente dos prontuários.

Howell et al. ${ }^{7}$ entrevistaram 785 indivíduos, onde $16 \%(\mathrm{n}=114)$ esperaram mais de três meses para buscar atendimento médico após seus primeiros sintomas, a idade média dos participantes da pesquisa foi de 58 anos. Rogers et al. ${ }^{9}$ entrevistaram 244 pessoas com câncer colorretal, onde $75 \%$ dos pacientes tinham mais de 50 anos e $64 \%$ foram diagnosticados em estágios avançados (III ou IV) da doença, o tempo desde a percepção dos sintomas iniciais até a busca de atendimento médico foi de dois a três meses. Foletto et al. ${ }^{11}$ entrevistaram 50 pacientes com câncer colorretal, a idade média foi de 56 anos e o tempo médio entre o início dos sintomas e a decisão de procurar um serviço de saúde foi de cinco a seis meses (177 dias). Felippu et al. ${ }^{12}$ entrevistaram 80 participantes diagnosticados com alguma malignidade de cabeça e pescoço, $94 \%(n=75)$ eram do sexo masculino, a idade média foi de 63 anos e a mediana do tempo entre o início dos sintomas e a busca de atendimento médico foi de dez meses. Valle et al. ${ }^{13}$ entrevistaram 101 pacientes diagnosticados com câncer colorretal ou de estômago, a idade média foi de 62 anos, $53 \%$ dos participantes eram do sexo masculino, a busca por atendimento médico ocorreu até um mês após o início dos sintomas em 67 casos $(66,3 \%)$, e em 34 (33,7\%) foram necessários mais de um mês para se encontrar um centro de saúde. Aguiar Jr. et al. ${ }^{14}$ entrevistaram 50 pacientes indígenas diagnosticados com algum tipo de tumor sólido, a média das idades foi de 54 anos e a maioria era do sexo feminino (58\%), o tempo médio entre o início dos sintomas e a busca por atendimento médico foi de nove meses. Le Campion et al. ${ }^{10}$ analisaram 121 prontuários de pacientes com idade superior a 18 anos, todos foram diagnosticados com algum tipo de câncer bucal, a idade média dos participantes foi de 64 anos, $67 \%$ destes eram do sexo masculino e o maior atraso estava relacionado ao paciente na busca pela primeira consulta médica após seus sintomas iniciais, sendo este tempo aproximadamente seis a sete meses (198 dias).

Na pesquisa realizada no HUAP, observou-se que, até o momento, os pacientes do gênero feminino buscam mais rapidamente o atendimento médico no início dos sintomas que o gênero masculino. Ainda, embora $48,72 \%$ dos pacientes buscassem o atendimento de imediato, em sua grande parte estavam no estágio avançado da doença. Foi verificado também, que a maior parte dos pacientes trabalhou em algum momento de sua carreira com produtos de limpezas e correlatos, e os mesmos tiveram sintomas parecidos como cansaço, dores no corpo e manchas roxas.

Como mencionado, assim que se manifestam os primeiros sintomas os pacientes buscam por ajuda médica e de acordo com o Art. $2^{\circ}$ da Lei $n^{\circ} 12.732$, de 22 de novembro de 2012

\begin{abstract}
"O paciente com neoplasia maligna tem direito de se submeter ao primeiro tratamento no Sistema Único de Saúde (SUS), no prazo de até 60 (sessenta) dias contados a partir do dia em que for firmado o diagnóstico em laudo patológico ou em prazo menor, conforme a necessidade terapêutica do caso registrada em prontuário único". ${ }^{16}$
\end{abstract}

Entretanto, foi analisado que haviam pacientes que buscaram atendimento rapidamente após os primeiros sintomas, porém tardando o encaminhamento ao especialista no HUAP, variando entre três meses a um ano, demonstrando assim, que embora exista um prazo máximo para o início do tratamento ainda é observada a demora entre alguns pacientes ultrapassando os 60 dias, podendo levar a um mau prognóstico da doença.

Howell et al. ${ }^{7}$ explicam que a demora pela busca médica pode ocorrer devido a muitos dos sintomas (como fadiga, linfadenopatia e dor óssea) serem comuns na população em geral. Por isso, podem ser justificados pelos pacientes como: consequência natural do envelhecimento, no caso da fadiga; a linfadenopatia pode ser atribuída a uma infecção localizada quando em torno do pescoço ou a uma hérnia, se localizada na virilha; e a dor óssea ao 'desgaste' relacionado com a idade. Enquanto Foletto et al. ${ }^{11}$ sugerem que o tempo prolongado não se deve apenas à falta de conhecimento dos pacientes, mas também a falta de tempo, o excesso de trabalho, o medo de um diagnóstico sem chances de cura e até o simples fato de considerar certos sintomas como normais. Ainda, Felippu et al. ${ }^{12}$ explicam que quanto mais avançado o diagnóstico, $55 \%$ dos pacientes foram diagnosticados no estágio IV da doença, mais difícil se torna um bom prognóstico. Valle et al. ${ }^{13}$ por vez, observaram que as mulheres tendem a aderir melhor a programas de saúde, como por exemplo, o programa de rastreio do câncer de mama, o que provavelmente é o fator que explica o aumento da incidência de neoplasias malignas, em geral, na população masculina

\section{Conclusão}

Sendo o atraso do paciente definido como o tempo decorrido desde a detecção dos primeiros sinais 
e sintomas até a procura pelo atendimento médico, ${ }^{10,15}$ conclui-se que, em todos os artigos selecionados e analisados e na pesquisa realizada no Hospital Universitário Antônio Pedro, os indivíduos tiveram um atraso significativo na procura de auxílio médico, de dois meses a até um ano. Grande parte dos pacientes tiveram diagnóstico em estágio avançado.

Essa demora pode estar relacionada à falta de conhecimento da população acerca dos primeiros sinais e sintomas de malignidades ou à associação desses sintomas a doenças não severas. Vale ressaltar que, há poucos trabalhos na área, apenas um artigo referente ao tema e relacionado a malignidades hematológicas, o que reforça a necessidade de pesquisas que delimitem programas de conscientização da população para busca por atendimento médico logo após os primeiros sinais, além de melhoria dos programas de rastreio desse tipo de neoplasia

\section{Comitê de Ética}

Comitê de Ética em Pesquisa da Faculdade de Medicina da UFF, Universidade Federal Fluminense. Endereço: Rua Marquês do Paraná 303, $4^{\circ}$ andar, prédio anexo ao HUAP. - Niterói - RJ. Telefone: +55 (21) 2629-9189. E-mail: etica.ret@id.uff.br. Número do Parecer: 3.285.266. Aprovado em 25 de Abril de 2019.

\section{Referências}

1. Souza MV, Christofoletti M, Streb AR, Duca GF Del. Quality of life and functional capacity during the treatment of hematologic neoplasms. Fisioter em Mov [Internet]. 2018 [cited 2020 Oct 19]; 31(0). Available from: http://www.scielo.br/scielo.php?script=sci_arttext\&pid=S0103$51502018000100227 \& \operatorname{lng}=$ en\&tlng=en

2. O Atlas do Câncer. Explore dados e percepções globais sobre câncer. [Internet]. [cited 2020 Oct 19]. Available from: www.cancer.org/canceratlas

3. INCA. Estimativa 2020: incidência de câncer no Brasil. INCA - Instituto Nacional de Câncer [Internet]. [cited 2020 Oct 19]. Available from: https:// www.inca.gov.br/publicacoes/livros/estimativa-2020-incidencia-de-cancerno-brasil

4. Mello CNH, Martins MLC, Chamone D, Pinto KO, Santos NO, Luciant MCS. Intervenções psicológicas realizadas na clínica onco-hematolótica: discussão acerca das possibilidades clínicas apresentadas na literatura. Psicologia Hospitalar [Internet]. 2007 [cited 2020 Oct 2019]; 5(1). Available from: http://pepsic.bvsalud.org/scielo.php?script=sci_arttext\&pid=S1677$74092007000100006 \& \operatorname{lng}=$ pt\&nrm $=$ iso

5. Ferlay J, Soerjomataram I, Dikshit R, Eser S, Mathers C, Rebelo M, Parkin DM, Forman D, Bray F. Cancer incidence and mortality worldwide: Sources, methods and major patterns in GLOBOCAN 2012 [Internet]. [cited 2020 Oct 19]. Available from: https://onlinelibrary.wiley.com/doi/full/10.1002/ ijc. 29210

6. Mcphail S, Elliss-Brookes L, Shelton J, Ives A, Greenslade M, Vernon $\mathrm{S}$, Morris E, Richards M. Emergency presentation of cancer and shortterm mortality. Br J Cancer [Internet]. 2013 [cited 2020 Oct 2019]; 109(8). Available from: https://www.ncbi.nlm.nih.gov/pmc/articles/PMC3798965/

7. Howell DA, Warburton F, Ramirez A-J, Roman E, Smith AG, Forbes LJL. Risk factors and time to symptomatic presentation in leukaemia, lymphoma and myeloma. Br J Cancer [Internet]. 2015 [cited 2020 Oct 2019]; 113(7).
Available from: https://www.ncbi.nlm.nih.gov/pmc/articles/PMC4651121/

8. Jacobsen MM, Silverstein SC, Quinn M, Waterston LB, Thomas CA, Benneyan JC, et al. Timeliness of access to lung cancer diagnosis and treatment: A scoping literature review. Lung Cancer [Internet]. 2017 [cited 2020 Oct 2019]; 112. Available from: http:/www.lungcancerjournal.info/ article/S0169500217304725/fulltext

9. Rogers HL, Siminoff LA, Longo DR, Thomson MD. Coping with Prediagnosis Symptoms of Colorectal Cancer: A Study of 244 Recently Diagnosed Individuals. Cancer nurs [Internet]. 2017 [cited 2020 Oct 2019]; 40(2). Available from: https://www.ncbi.nlm.nih.gov/pmc/articles/ PMC5045736/

10. Le Campion ACOV, Santos KCB, Carmo ES, Silva Júnior FF da, Peixoto FB, Ribeiro CMB, Gonçalves LS, Ferreira SMS. Caracterização do atraso no diagnóstico do câncer de boca e orofaringe em dois centros de referência. Cad Saúde Coletiva [Internet]. 2016 [cited 2020 Oct 19]; 24(2). Available from: http://www.scielo.br/scielo.php?script=sci_arttext\&pid=S1414-462X $2016000200178 \& \operatorname{lng}=$ en\&nrm=iso\&tlng=pt.

11. Foletto EF, Jackisch SE, Dotto ML, Severo C, Pappen E, Valim ARM, Renner JDP, Possuelo LG. Therapeutic itinerary of colorectal cancer patients treated in the state of Rio Grande do Sul. J Coloproctology. 2016 [cited 2020 Oct 19]; 36(2).

12. Felippu AWD, Freire EC, de Arruda Silva R, Guimarães AV, Dedivitis RA. Impact of delay in the diagnosis and treatment of head and neck cancer. Braz J Otorhinolaryngol [Internet]. 2016 [cited 2020 Oct 19];82(2).

13. Valle TD, Turrini RNT, De Brito Poveda V. Intervening factors for the initiation of treatment of patients with stomach and colorectal cancer. Rev Lat Am Enfermagem [Internet]. 2017 [cited 2020 Oct 19]; 25. Available from: http://www.scielo.br/scielo.php?script=sci_arttext\&pid=S0104$11692017000100333 \& \operatorname{lng}=$ en\&nrm $=$ iso

14. Aguiar PN, Stock GT, Lopes GL, Almeida MS, Tadokoro H, Gutierres BS, RodriAgues DA. Disparities in cancer epidemiology and care delivery among Brazilian indigenous populations. Einstein (Sao Paulo) [Internet] 2016 [cited 2020 Oct 19];14(3). Available from: http://www.scielo.br/scielo. php? script $=$ sci_arttext\&pid $=\mathrm{S} 1679-45082016000300330 \& \operatorname{lng}=\mathrm{en} \& \mathrm{nrm}=\mathrm{is}$ o\&tlng=en

15. Güneri P, Epstein JB. Late stage diagnosis of oral cancer: Components and possible solutions. Oral Oncology [Internet]. 2014 [cited 2020 Oct 19]; 50(12). Available from: https://pubmed.ncbi.nlm.nih.gov/25255960/

16. L12732 [Internet]. [cited 2020 Oct 19]. Available from: http://www. planalto.gov.br/ccivil_03/_ato2011-2014/2012/lei/112732.htm 
Questionário aplicado ao paciente:

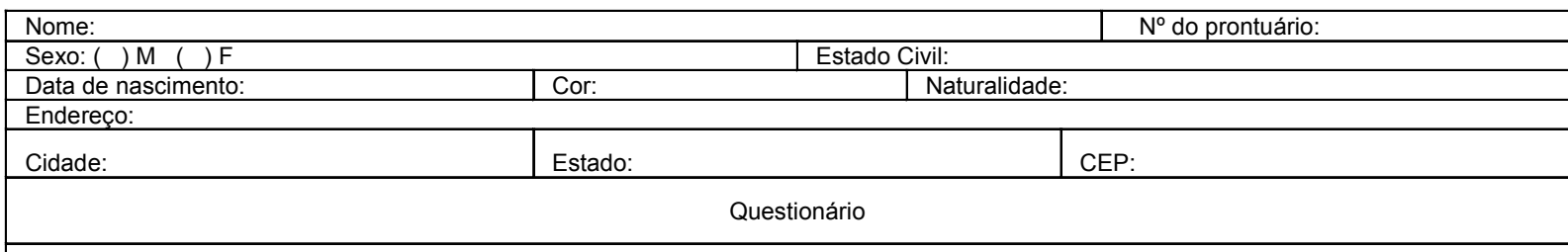

1. Em qual profissão trabalhou a maior parte da sua vida?

2. Fazia exames periódicos a pedido de seu serviço?

( ) Não;

( ) Sim, De quanto em quanto tempo?

3. Tem casos de câncer em sua família?

( ) Não;

( ) Sim, Quem (exemplo: pais, avós, etc)?

4. Se a resposta da pergunta 3 foi sim, qual tipo de câncer?

( ) Algum parecido com o que eu tenho;

( ) Outro (diga qual se souber)

5. Como descobriu seu diagnóstico?

( ) Estava com uma dor e fui verificar o que era:

( ) Em um exame de rotina do serviço;

( ) Em um exame de rotina pedido por mim;

( ) Perdi muito peso e fui ver o que era;

( ) Outro

6. Quais sintomas tinha antes de procurar um atendimento?

7. Você buscou atendimento médico assim que sentiu estas dores ou as 'deixou de lado' por algum tempo (se 'deixou de lado', diga por quanto tempo)?

8. Procurou outros atendimentos antes de chegar ao HUAP?

( ) Não, vim direto ao HUAP;

( ) Sim.

9. Se a resposta da pergunta 8 foi sim diga qual atendimento procurou:

( ) Unidade Básica de Saúde - UBS/PSF;

( ) Atendimento médico particular;

( ) Outro hospital;

( ) Outro.

10. Se respondeu à questão 9 diga, quanto tempo em média levou para ser encaminhado deste outro serviço até o HUAP?

11. Recebeu alguma confirmação de diagnóstico ou tratamento neste outro serviço?

12. Diga, se souber, aproximadamente em qual estágio teve seu diagnóstico?

( ) Inicial

( ) Tardio

( ) Muito avançado

( ) Outro

13. Fuma?

( ) Nunca.

( ) Já fumei.

- Quanto tempo?

- Parou há quanto tempo?

- Lembra a quantidade?

( ) Fumo

- Quanto tempo?

- Lembra a quantidade?

14. Bebe?

( ) Nunca;

( ) Já bebi;

- Quanto tempo?

- Parou há quanto tempo?

- Lembra a quantidade?

( ) Bebo.

- Quanto tempo?

- Lembra a quantidade? 